\title{
The Burr X Exponentiated Weibull Model: Characterizations, Mathematical Properties and Applications to Failure and Survival Times Data
}

\author{
Mohamed G. Khalil \\ Department of Statistics, Mathematics and Insurance, \\ Benha University, Benha, Egypt. \\ hndaoy@gmail.com \\ G. G. Hamedani \\ Department of Mathematics, Statistics and Computer Science, \\ Marquette University, USA. \\ gholamhoss.hamedani@marquette.edu \\ Haitham M. Yousof \\ Department of Statistics, Mathematics and Insurance, \\ Benha University, Benha, Egypt. \\ haitham.yousof@fcom.bu.edu.eg
}

\begin{abstract}
In this article, we introduce a new three-parameter lifetime model called the Burr X exponentiated Weibull model. The major justification for the practicality of the new lifetime model is based on the wider use of the exponentiated Weibull and Weibull models. We are motivated to propose this new lifetime model because it exhibits increasing, decreasing, bathtub, $\mathbf{J}$ shaped and constant hazard rates. The new lifetime model can be viewed as a mixture of the exponentiated Weibull distribution. It can also be viewed as a suitable model for fitting the right skewed, symmetric, left skewed and unimodal data. We provide a comprehensive account of some of its statistical properties. Some useful characterization results are presented. The maximum likelihood method is used to estimate the model parameters. We prove empirically the importance and flexibility of the new model in modeling two types of lifetime data. The proposed model is a better fit than the Poisson Topp Leone-Weibull, the Marshall Olkin extended-Weibull, gamma-Weibull , Kumaraswamy-Weibull , Weibull-Fréchet, beta-Weibull, transmuted modified-Weibull, Kumaraswamy transmuted- Weibull, modified beta-Weibull, Mcdonald-Weibull and transmuted exponentiated generalized-Weibull models so it is a good alternative to these models in modeling aircraft windshield data as well as the new lifetime model is much better than the Weibull-Weibull, odd WeibullWeibull, Weibull Log-Weibull, the gamma exponentiated-exponential and exponential exponential-geometric models so it is a good alternative to these models in modeling the survival times of Guinea pigs. We hope that the new distribution will attract wider applications in reliability, engineering and other areas of research.
\end{abstract}

Keywords: Burr X family, Order Statistics, Exponentiated Weibull, Maximum Likelihood Estimation, Characterizations, Quantile function, Moments, Generating Function.

\section{Introduction}

It is known that the Weibull distribution has been the most popular distribution for modeling lifetimes (see Murthy et al., 2004 and Rinne, 2009) and has been extensively used for modeling data in engineering, reliability and biological researches. The major weakness of this distribution is its inability to accommodating nonmonotone hazard rates. This has led to the need of exploring more generalizing of this model. The first generalization allowing for nonmonotone hazard rates is the exponentiated Weibull (EW) model (see Mudholkar and Srivastava (1993) and Mudholkar et al. (1995)). The goal of this paper is to introduce a new extremely flexible version of the EW model. 
A random variable (rv) $Z$ is said to have the $\mathrm{EW}$ distribution if its probability density function (pdf) and cumulative distribution function (cdf) are given by

$$
\begin{aligned}
& g_{E W}(z ; \alpha, \beta)=\alpha \beta z^{\beta-1} \exp \left(-z^{\beta}\right)\left[1-\exp \left(-z^{\beta}\right)\right]^{\alpha-1} \text { and } G_{E W}(z ; \alpha, \beta) \\
& \quad=\left[1-\exp \left(-z^{\beta}\right)\right]^{\alpha},
\end{aligned}
$$

respectively, for $z>0, \alpha>0$ and $\beta>0$. Yousof et al. (2017) introduced a flexible family of distributions called Burr $\mathrm{X}$ generator $(\mathrm{BrX}-\mathrm{G})$ with

$$
F(x ; \theta, \xi)=\left(1-\exp \left\{-[G(x ; \xi) / \bar{G}(x ; \xi)]^{2}\right\}\right)^{\theta}, x \in R,
$$

and pdf

$$
\begin{aligned}
& f(x ; \theta, \xi)=2 \theta g(x ; \xi) G(x ; \xi) \bar{G}(x ; \xi)^{-3} \exp \left\{-[G(x ; \xi) / \bar{G}(x ; \xi)]^{2}\right\} \\
& \times\left(1-\exp \left\{-[G(x ; \xi) / \bar{G}(x ; \xi)]^{2}\right\}\right)^{\theta-1}, x \in R .
\end{aligned}
$$

To this end we will use the $\mathrm{BrX}-\mathrm{G}$ for generating the new extreme flexible version of the $\mathrm{EW}$ model.

This paper is organized as follows. In Section 2, we define the new distribution. Section 3 deals with some characterizations of the new model. we derive some of its mathematical properties in Section 4. The maximum likelihood method is presented in Section 5. In Section 6, we illustrate the importance of the new model by means of two applications to real data sets. The paper is concluded in Section 7.

\section{The new model and its justification}

By inserting $G_{E W}(x ; \alpha, \beta)$ in (1) we obtain the cdf of the Burr X exponentiated Weibull (BrXEW) model as

$$
F(x ; \theta, \alpha, \beta)=\left[1-\exp \left(-\left\{\frac{\left[1-\exp \left(-x^{\beta}\right)\right]^{\alpha}}{1-\left[1-\exp \left(-x^{\beta}\right)\right]^{\alpha}}\right\}^{2}\right)\right]^{\theta}, x \geq 0 .
$$

The corresponding pdf is

$$
\begin{gathered}
=\frac{2 \theta \alpha \beta x^{\beta-1} \exp \left(-x^{\beta}\right)\left[1-\exp \left(-x^{\beta}\right)\right]^{2 \alpha-1}}{\left\{1-\left[1-\exp \left(-x^{\beta}\right)\right]^{\alpha}\right\}^{3}} \exp \left(-\left\{\frac{\left[1-\exp \left(-x^{\beta}\right)\right]^{\alpha}}{1-\left[1-\exp \left(-x^{\beta}\right)\right]^{\alpha}}\right\}^{2}\right) \\
\times\left[1-\exp \left(-\left\{\frac{\left[1-\exp \left(-x^{\beta}\right)\right]^{\alpha}}{1-\left[1-\exp \left(-x^{\beta}\right)\right]^{\alpha}}\right)^{2}\right)\right]^{\theta-1}, x>0 .
\end{gathered}
$$

$A$

Now, we provide a very useful linear representation for the BrXEW density function. If $|z|<1$ and $b>0$ is a real non-integer, the following power series holds 


$$
(1-z)^{b-1}=\sum_{i=0}^{\infty}(-1)^{i} \Gamma(b) z^{i} /[i ! \Gamma(b-i)] .
$$

Applying (5) to the term $\mathrm{A}$ in (4) we have

$$
\begin{aligned}
f(x)= & \frac{2 \theta \alpha \beta x^{\beta-1} \exp \left(-x^{\beta}\right)\left[1-\exp \left(-x^{\beta}\right)\right]^{2 \alpha-1}}{\left\{\left[1-\exp \left(-x^{\beta}\right)\right]^{\alpha}\right\}^{3}} \sum_{i=0}^{\infty} \frac{(-1)^{i} \Gamma(\theta)}{i ! \Gamma(\theta-i)} \\
& \times \underbrace{\exp \left(-(i+1)\left\{\frac{\left[1-\exp \left(-x^{\beta}\right)\right]^{\alpha}}{1-\left[1-\exp \left(-x^{\beta}\right)\right]^{\alpha}}\right\}^{2}\right)}, x>0 .
\end{aligned}
$$

Applying the power series to the term $B$ in (6) we have

$$
\begin{aligned}
f(x)=\sum_{i, j=0}^{\infty} \frac{2 \theta \alpha \beta x^{\beta-1} \exp \left(-x^{\beta}\right)(-1)^{i+j}(i+1)^{j} \Gamma(\theta)}{i ! j ! \Gamma(\theta-i)\left[1-\exp \left(-x^{\beta}\right)\right]^{1-\alpha}} \\
\times \underbrace{\frac{\left\{\left[1-\exp \left(-x^{\beta}\right)\right]^{\alpha}\right\}^{2 j+1}}{\left\{1-\left[1-\exp \left(-x^{\beta}\right)\right]^{\alpha}\right\}^{2 j+3}}} .
\end{aligned}
$$

Consider the series expansion

$$
\text { C }
$$

$$
(1-z)^{-b}=\sum_{k=0}^{\infty} \Gamma(b+k) z^{k} /[k ! \Gamma(b)],|z|<1, b>0 .
$$

Applying the expansion (8) to (7) to $\mathrm{C}$ in (7) we arrive at

where

$$
f(x)=\sum_{j, k=0}^{\infty} \delta_{j, k} \pi_{(2 j+k+2) \alpha}(x)
$$

$$
\delta_{j, k}=\frac{2 \theta(-1)^{j} \Gamma(\theta) \Gamma(2 j+k+3)}{j ! k ! \Gamma(2 j+3)[(2 j+k+2) \alpha]} \sum_{i=0}^{\infty} \frac{(-1)^{i}(i+1)^{j}}{i ! \Gamma(\theta-i)},
$$

and $\pi_{(2 j+k+2) \alpha}(x)$ is the cdf of the EW model with power parameter $(2 j+k+2) \alpha$.

Equation (5) reveals that the density of $X$ can be expressed as a linear mixture of EW densities. So, several mathematical properties of the new family can be obtained from those of the exp-Li distribution. Similarly, the cdf of the BrXEW model can also be expressed as a mixture of EW cdfs given by

$$
F(x)=\sum_{j, k=0}^{\infty} \delta_{j, k} \underbrace{\left[1-\exp \left(-x^{\beta}\right)\right]^{(2 j+k+2) \alpha}}_{\Pi_{(2 j+k+2) \alpha}(x)},
$$

where $\Pi_{(2 j+k+2) \alpha}(x)$ is the cdf of the EW model with power parameter $(2 j+k+2) \alpha$.

The justification for the practicality of the BrXEW lifetime model is based on the wider use of the EW and W models. We are also motivated to introduce the BrXEW lifetime model since it exhibits increasing, decreasing, bathtub, J shaped, and constant hazard rates as illustrated in Figure 2 (b1 to b5, respectively). We mentioned before that the BrXEW lifetime model can be viewed as a mixture of the EW distribution. It can be considered as a suitable model for fitting 
the right skewed, symmetric, left skewed and unimodal data. The proposed BrXEW lifetime model is a much better fit than the Poisson Topp Leone-Weibull, the Marshall Olkin extendedWeibull, gamma-Weibull, Kumaraswamy-Weibull, Weibull-Fréchet, beta-Weibull, transmuted modified-Weibull, Kumaraswamy transmuted- Weibull, modified beta-Weibull, McdonaldWeibull and transmuted exponentiated generalized-Weibull models, so the new lifetime model is a good alternative to these models in modeling aircraft windshield data. It is also a much better fit than the Weibull-Weibull, odd Weibull-Weibull, Weibull Log-Weibull, the gamma exponentiated-exponential and exponential exponential-geometric models, so it is a good alternative to these models in modeling the survival times of Guinea pigs.
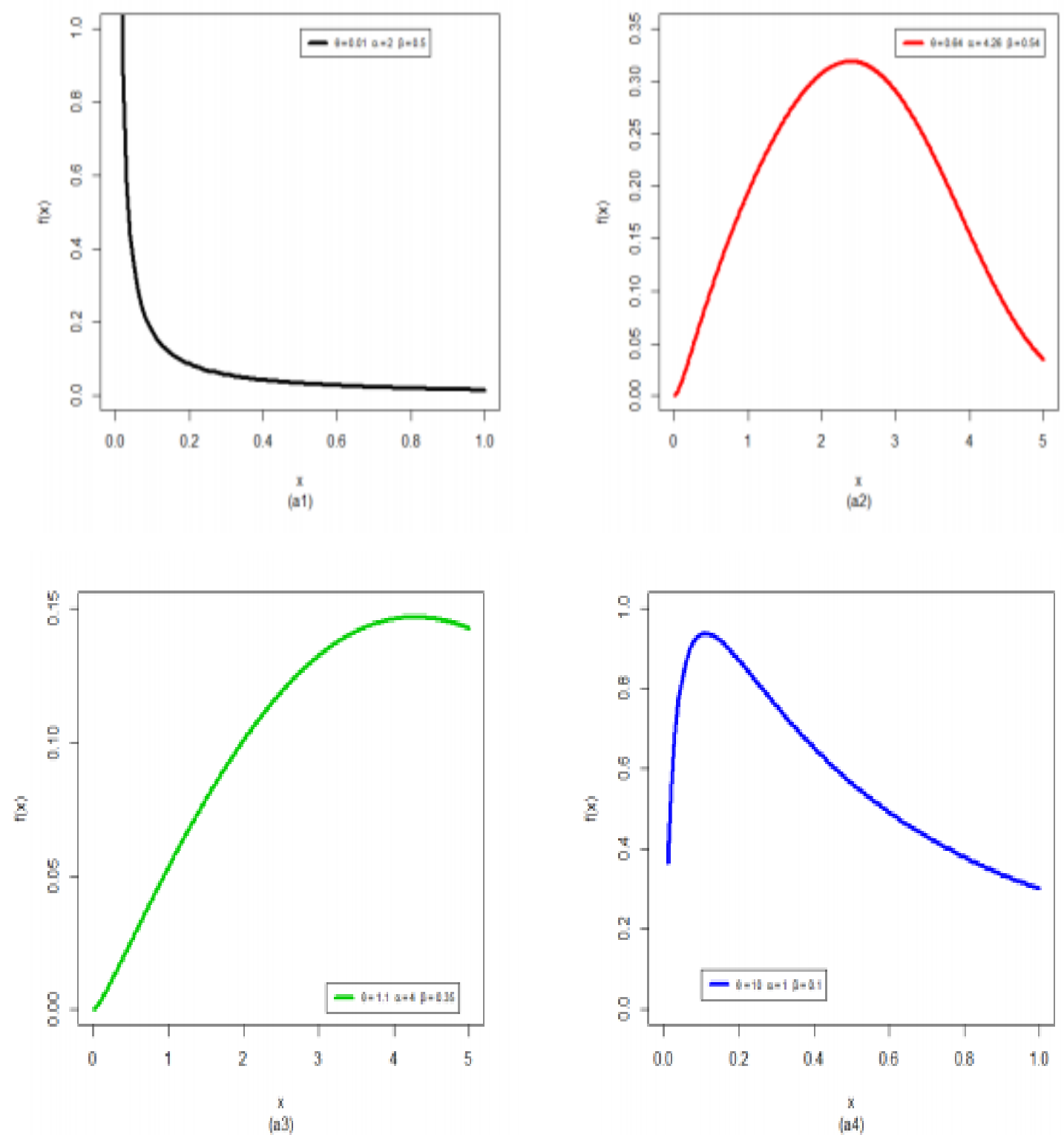

Figure 1: Plots of the BrXEW pdf. 

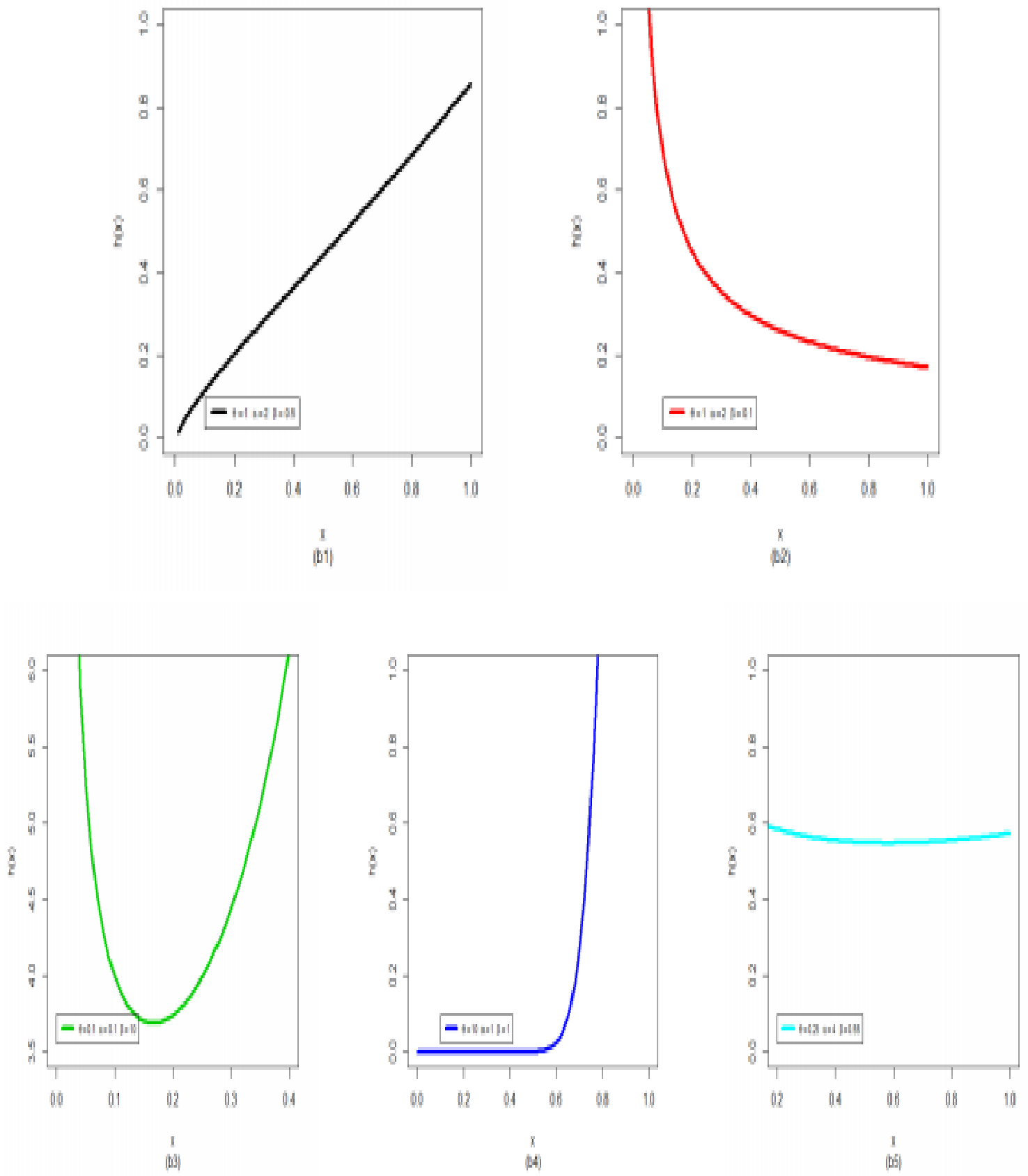

Figure 2: Plots of the BrXEW hrf.

\section{Characterizations results}

This section is devoted to the characterizations of the BrXEW distribution in different directions: ( $i$ ) based on the ratio of two truncated moments; (ii) in terms of the hazard function; ( $i i i$ ) in terms of the reverse hazard function and (iv) based on the conditional expectation of certain function of the random variable. Note that $(i)$ can be employed also when the cdf does not have a closed form. We would also like to mention that due to the nature 
of BrXEW distribution, our characterizations may be the only possible ones. We present our characterizations $(i)-(i v)$ in four subsections.

\subsection{Characterizations based on two truncated moments}

This subsection deals with the characterizations of BrXEW distribution based on the ratio of two truncated moments. Our first characterization employs a theorem due to Glänzel (1987), see Theorem 1 (see Hamedani et al. (2018) and Hamedani et al. (2019)). The result, however, holds also when the interval $H$ is not closed, since the condition of the Theorem is on the interior of $H$.

Proposition 3.1. Let $X: \Omega \rightarrow(0, \infty)$ be a continuous rv and let

$$
q_{2}(x)=\frac{\left(1-\left[1-\exp \left(-x^{\beta}\right)\right]^{\alpha}\right)^{3}}{\exp \left(-\left\{\frac{\left[1-\exp \left(-x^{\beta}\right)\right]^{\alpha}}{1-\left[1-\exp \left(-x^{\beta}\right)\right]^{\alpha}}\right\}^{2}\right)\left[1-\exp \left(-\left\{\frac{\left[1-\exp \left(-x^{\beta}\right)\right]^{\alpha}}{1-\left[1-\exp \left(-x^{\beta}\right)\right]^{\alpha}}\right\}^{2}\right)\right]^{\theta-1} .}
$$

and $q_{1}(x)=q_{2}(x)\left[1-e^{-x^{\beta}}\right]^{-\alpha}$ for $x>0$. The rv $X$ has pdf (4) if and only if the function $\eta$ defined in Theorem 1 is of the form

$$
\eta(x)=\frac{1}{2}\left\{1+\left[1-\exp \left(-x^{\beta}\right)\right]^{\alpha}\right\}, \quad x>0 .
$$

Proof. Suppose the rv $X$ has pdf (4), then

and

$$
(1-F(x)) E\left[q_{1}(X) \mid X \geq x\right]=2 \theta\left\{1-\left[1-\exp \left(-x^{\beta}\right)\right]^{\alpha}\right\}, \quad x>0
$$

$$
(1-F(x)) E\left[q_{2}(X) \mid X \geq x\right]=\theta\left\{1-\left[1-\exp \left(-x^{\beta}\right)\right]^{2 \alpha}\right\}, \quad x>0
$$

Further,

$$
\eta(x) q_{1}(x)-q_{2}(x)=\frac{q_{2}(x)}{2}\left\{\frac{1-\left[1-\exp \left(-x^{\beta}\right)\right]^{\alpha}}{\left[1-\exp \left(-x^{\beta}\right)\right]^{\alpha}}\right\}>0, \text { for } x>0 .
$$

Conversely, if $\eta$ is of the above form, then

$$
s^{\prime}(x)=\frac{\eta^{\prime}(x) q_{1}(x)}{\eta(x) q_{1}(x)-q_{2}(x)}=\frac{\alpha \beta x^{\beta-1} \exp \left(-x^{\beta}\right)\left[1-\exp \left(-x^{\beta}\right)\right]^{\alpha-1}}{1-\left[1-\exp \left(-x^{\beta}\right)\right]^{\alpha}}, x>0
$$

and consequently $s(x)=-\log \left\{1-\left[1-\exp \left(-x^{\beta}\right)\right]^{\alpha}\right\}, \quad x>0$. Now, according to Theorem 1, $X$ has density (4).

Corollary 3.1. Let $X: \Omega \rightarrow(0, \infty)$ be a continuous rv and let $q_{2}(x)$ be as in Proposition 3.1. The rv $X$ has pdf (4) if and only if there exist functions $q_{1}$ and $\eta$ defined in Theorem 1 satisfying the following differential equation

$$
\frac{\eta^{\prime}(x) q_{1}(x)}{\eta(x) q_{1}(x)-q_{2}(x)}=\frac{\alpha \beta x^{\beta-1} \exp \left(-x^{\beta}\right)\left[1-\exp \left(-x^{\beta}\right)\right]^{\alpha-1}}{1-\left[1-\exp \left(-x^{\beta}\right)\right]^{\alpha}}, \quad x>0 .
$$

Corollary 3.2. The general solution of the differential equation in Corollary A.1 is 


$$
\begin{aligned}
& \eta(x)=\left\{1-\left[1-\exp \left(-x^{\beta}\right)\right]^{\alpha}\right\}^{-1} \\
& \times\left[-\int \alpha \beta x^{\beta-1} \exp \left(-x^{\beta}\right)\left[1-\exp \left(-x^{\beta}\right)\right]^{\alpha-1}\left(q_{1}(x)\right)^{-1} q_{2}(x) d x+D\right],
\end{aligned}
$$

where $D$ is a constant. We like to point out that one set of functions satisfying the above differential equation is given in Proposition 3.1 with $D=\frac{1}{2}$.

Clearly, there are other triplets $\left(q_{1}, q_{2}, \eta\right)$ which satisfy conditions of Theorem1.

\subsection{Characterization in terms of hazard function}

The hazard function, $h_{F}$, of a twice differentiable distribution function, $F$, satisfies the following first order differential equation

$$
\frac{f^{\prime}(x)}{f(x)}=\frac{h_{F}^{\prime}(x)}{h_{F}(x)}-h_{F}(x) .
$$

It should be mentioned that for many univariate continuous distributions, the above equation is the only differential equation available in terms of the hazard function. In this subsection we present non-trivial characterizations of BrXEW distribution, for $\theta=1$, in terms of the hazard function.

Proposition A.2. Let $X: \Omega \rightarrow(0, \infty)$ be a continuous random variable. The rv $X$ has pdf (4) if and only if its hazard function $h_{F}(x)$ satisfies the following differential equation

$$
h_{F}^{\prime}(x)+\beta x^{\beta-1} h_{F}(x)=2 \alpha \beta \exp \left(-x^{\beta}\right) \frac{d}{d x}\left\{\frac{x^{\beta-1}\left[1-\exp \left(-x^{\beta}\right)\right]^{2 \alpha-1}}{\left(1-\left[1-\exp \left(-x^{\beta}\right)\right]^{\alpha}\right)^{3}}\right\}, x>0 .
$$

Proof. If $X$ has pdf (4), then clearly the above differential equation holds. If the differential equation holds, then

$$
\frac{d}{d x}\left\{e^{x^{\beta}} h_{F}(x)\right\}=2 \alpha \beta \frac{d}{d x}\left\{\frac{x^{\beta-1}\left[1-\exp \left(-x^{\beta}\right)\right]^{2 \alpha-1}}{\left(1-\left[1-\exp \left(-x^{\beta}\right)\right]^{\alpha}\right)^{3}}\right\}
$$

from which we arrive at the hazard function of (4) when $\theta=1$.

3.3 Characterization in terms of the reverse hazard function

The reverse hazard function, $r_{F}$, of a twice differentiable distribution function, $F$, is defined as

$$
r_{F}(x)=\frac{f(x)}{F(x)}, \quad x \in \text { support of } F \text {. }
$$

In this subsection we present a characterization of BrXEW distribution in terms of the reverse hazard function.

Proposition 3.3. Let $X: \Omega \rightarrow(0, \infty)$ be a continuous random variable. The rv $X$ has pdf (4) if and only if its reverse hazard function $r_{F}(x)$ satisfies the following differential equation $r_{F}^{\prime}(x)+\beta x^{\beta-1} r_{F}(x)=$ 


$$
2 \theta \alpha \beta e^{-x^{\beta}} \frac{d}{d x}\left\{\frac{\frac{x^{\beta-1} \exp \left(-x^{\beta}\right)}{\left[1-\exp \left(-x^{\beta}\right)\right]^{1-2 \alpha}} \exp \left(-\left\{\frac{\left[1-\exp \left(-x^{\beta}\right)\right]^{\alpha}}{1-\left[1-e^{-x^{\beta}}\right]^{\alpha}}\right\}^{2}\right)}{\left(1-\left[1-\exp \left(-x^{\beta}\right)\right]^{\alpha}\right)^{3}\left[1-\exp \left(-\left\{\frac{\left[1-\exp \left(-x^{\beta}\right)\right]^{\alpha}}{1-\left[1-\exp \left(-x^{\beta}\right)\right]^{\alpha}}\right\}^{2}\right)\right]}, x>0 .\right.
$$

Proof. Is similar to that of Proposition 3.2.

\subsection{Characterization based on the conditional expectation of certain function of the random variable}

In this subsection we employ a single function $\psi$ (or $\psi_{1}$ ) of $X$ and characterize the distribution of $X$ in terms of the truncated moment of $\psi(X)$ (or $\psi_{1}(X)$ ). The following propositions have already appeared in Hamedani's previous work (2013), so we will just state them here which can be used to characterize BrXEW distribution.

Proposition 3.4. Let $X: \Omega \rightarrow(e, f)$ be a continuous rv with $c d f F$. Let $\psi(x)$ be a differentiable function on $(e, f)$ with $\lim _{x \rightarrow e^{+}} \psi(x)=1$. Then for $\delta \neq 1$,

if and only if

$$
E[\psi(X) \mid X \geq x]=\delta \psi(x), \quad x \in(e, f)
$$

$$
\psi(x)=(1-F(x))^{\frac{1}{\delta}-1}, \quad x \in(e, f)
$$

Proposition 3.5. Let $X: \Omega \rightarrow(e, f)$ be a continuous rv with $c d f \quad F$. Let $\psi_{1}(x)$ be a differentiable function on $(e, f)$ with $\lim _{x \rightarrow f^{-}} \psi_{1}(x)=1$. Then for $\delta_{1} \neq 1$,

$$
E\left[\psi_{1}(X) \mid X \leq x\right]=\delta_{1} \psi_{1}(x), \quad x \in(e, f) \text { implies } \psi_{1}(x)=(F(x))^{\frac{1}{\delta_{1}}-1} . \quad x \in(e, f) .
$$

\section{Remarks 3.1.}

(A) For $(e, f)=(0, \infty), \quad \theta=1$,

$$
\psi(x)=\exp \left(-\left\{\frac{\left[1-\exp \left(-x^{\beta}\right)\right]^{\alpha}}{1-\left[1-\exp \left(-x^{\beta}\right)\right]^{\alpha}}\right\}^{2}\right)
$$

and $\delta=\frac{1}{2}$, Proposition 3.4 provides a characterization of BrXEW distribution.

(B) For $(e, f)=(0, \infty)$,

$$
\psi_{1}(x)=1-\exp \left(-\left\{\frac{\left[1-\exp \left(-x^{\beta}\right)\right]^{\alpha}}{1-\left[1-\exp \left(-x^{\beta}\right)\right]^{\alpha}}\right\}^{2}\right)
$$

and $\delta_{1}=\frac{\theta}{1+\theta}$. Proposition 3.5 provides a characterization of BrXEW distribution.

\section{Mathematical properties \\ 4.1 Moments}

The $r$ th ordinary moment of $X$ is given by 


$$
\mu_{r}^{\prime}=E\left(X^{r}\right)=\int_{-\infty}^{\infty} x^{r} f(x) d x=\Gamma\left(1+\frac{r}{\beta}\right) \sum_{j, k, m=0}^{\infty} q_{j, k, m}^{((2 j+k+2) \alpha, r)}, \forall r>-\beta,
$$

where

and

$$
q_{j, k, m}^{((2 j+k+2) \alpha, r)}=\delta_{j, k} v_{m}^{((2 j+k+2) \alpha, r)}
$$

$$
v_{a_{1}}^{\left(a_{2}, a_{3}\right)}=a_{2}(-1)^{a_{1}}\left(\begin{array}{c}
a_{2}-1 \\
a_{1}
\end{array}\right) /\left(a_{1}+1\right)^{\left(a_{3}+\beta\right) / \beta} .
$$

Setting $r=1$ in (11), we have the mean of $X$.

\subsection{Generating function}

Using the series expansion

$$
(1-z)^{a}=\sum_{i=0}^{\infty}\left(\begin{array}{l}
a \\
i
\end{array}\right)(-z)^{i} \text { for }|z|<1,
$$

one can expand $g_{E W}(x ;(2 j+k+2) \alpha, \beta)$ as

$$
g_{E W}(x ;(2 j+k+2) \alpha, \beta)=[(2 j+k+2) \alpha] \sum_{m=0}^{\infty}\left(\begin{array}{c}
k \\
m
\end{array}\right)\left[(-1)^{m} /(m+1)\right] g_{\left[(m+1)^{1 / \beta}\right]}(x),
$$

where $g_{\left[(m+1)^{1 / \beta}\right]}(x)$ denotes the pdf of the one-parameter Weibull distribution. So, whenever possible, $\pi_{(2 j+k+2) \alpha}(x)$ can be used to derive moment generating function of the BrXEW distribution from those of the one-parameter Weibull distribution. Let $p \Psi_{q}(\cdot)$ is the complex parameter Wright generalized hypergeometric (WGH) function with $p$ numerator and $q$ denominator parameters (Kilbas et al., 2006, Equation (1.9)) defined by the series

$$
p \Psi_{q}\left[\begin{array}{l}
\left(\alpha_{1}, A_{1}\right), \ldots,\left(\alpha_{p}, A_{p}\right) \\
\left(\beta_{1}, B_{1}\right), \ldots,\left(\beta_{q}, B_{q}\right)
\end{array}\right]=\sum_{n=0}^{\infty} \frac{\prod_{j=1}^{p} \Gamma\left(\alpha_{j}+A_{j} n\right)}{\prod_{j=1}^{q} \Gamma\left(\beta_{j}+B_{j} n\right)} \frac{z^{n}}{n !}
$$

Then, following similar algebraic developments of Nadarajah et al. (2013), we can write the mgf of $g_{E W}(x ;(2 j+k+2) \alpha, \beta)$ say $M_{Z}(t ;(2 j+k+2) \alpha, \beta)$, as

$$
\begin{aligned}
& M_{Z}(t ;(2 j+k+2) \alpha, \beta)=[(2 j+k+2) \alpha] \sum_{m=0}^{\infty} \frac{(-1)^{m}}{m+1}\left(\begin{array}{c}
(2 j+k+2) \alpha-1 \\
m
\end{array}\right) \\
& \times_{1} \Psi_{0}\left[\left(1,-\beta^{-1}\right) ;(m+1)^{1 / \beta} t\right],
\end{aligned}
$$

Hence, the mgf of the BrXEW model follows from (9) as

$$
M_{X}(t)=\sum_{m=0}^{\infty} a_{m} \Psi_{0}\left[\begin{array}{c}
\left(1,-\beta^{-1}\right) \\
-
\end{array}(m+1)^{1 / \beta} t\right]
$$

where $a_{m}=\left[(-1)^{m} /(m+1)\right] \sum_{j, k}^{\infty} \delta_{j, k}[(2 j+k+2) \alpha]\left(\begin{array}{c}(2 j+k+2) \alpha-1 \\ m\end{array}\right)$. The above equation $M_{X}(t)$ can be easily evaluated by scripts of the Matlab, Maple and Mathematica platforms.

\subsection{Incomplete moments}

The $s^{\text {th }}$ incomplete moment, say $\phi_{s}(t)=\int_{-\infty}^{t} x^{s} f(x) d x$, of $X$ can be expressed from (10) as 


$$
\begin{gathered}
\phi_{s}(t)=\sum_{j, k=0}^{\infty} \delta_{j, k} \int_{-\infty}^{t} x^{s} \pi_{(2 j+k+2) \alpha}(x) d x \\
=\gamma\left(1+\frac{s}{\beta},\left(\frac{1}{t}\right)^{\beta}\right) \sum_{j, k, m=0}^{\infty} q_{j, k, m}^{((2 j+k+2) \alpha, s)}, \forall s>-\beta .
\end{gathered}
$$

The first incomplete moment is obtained by setting $s=1$ in $\phi_{s}(t)$.

\subsection{Probability weighted moments}

The $(s, r)^{t h}$ PWM of $X$ following the BrXEW model, say $\rho_{s, r}$, is formally defined by

$$
\rho_{s, r}=E\left\{X^{s} F(X)^{r}\right\}=\int_{-\infty}^{\infty} x^{s} F(x)^{r} f(x) d x .
$$

Using equations (3), (4), (9) and (10) we can write

where

$$
f(x) F(x)^{r}=\sum_{j, k=0}^{\infty} a_{j, k} \pi_{(2 j+k+2) \alpha}(x),
$$

$$
a_{j, k}=\frac{2 \theta(-1)^{j} \Gamma(2 j+k+3)}{j ! k ! \Gamma(2 j+3)[(2 j+k+2) \alpha]} \sum_{i=0}^{\infty}(-1)^{i}(i+1)^{j}\left(\begin{array}{c}
\theta(r+1)-1 \\
i
\end{array}\right) .
$$

Then, the $(s, r)^{\text {th }}$ PWM of $X$ can be expressed as

where

$$
\rho_{s, r}=\Gamma\left(1+\frac{s}{\beta}\right) \sum_{j, k, m=0}^{\infty} a_{j, k, m}^{((2 j+k+2) \alpha, s)}, \forall s>-\beta,
$$

$$
a_{j, k, m}^{((2 j+k+2) \alpha, s)}=a_{j, k} v_{m}^{((2 j+k+2) \alpha, s)}
$$

\subsection{Residual and reversed residual life}

The $n^{\text {th }}$ moment of the residual life, say $m_{n}(t)=E\left[(X-t)^{n} \mid X>t\right], n=1,2$, uniquely determine $F(x)$. The $n$th moment of the residual life of $X$ is given by $m_{n}(t)=$ $[1-F(t)]^{-1} \int_{t}^{\infty}(x-t)^{n} d F(x)$. Therefore

$$
m_{n}(t)=\gamma\left(1+\frac{n}{\beta},\left(\frac{1}{t}\right)^{\beta}\right)[1-F(t)]^{-1} \sum_{j, k, m=0}^{\infty} \sum_{r=0}^{n} \zeta_{j, k, m, r}^{((2 j+k+2) \alpha, n)}, \forall n>-\beta,
$$

where

$$
\zeta_{j, k, m}^{((2 j+k+2) \alpha, n)}=\delta_{j, k} v_{m}^{((2 j+k+2) \alpha, n)}\left(\begin{array}{l}
n \\
r
\end{array}\right)(-t)^{n-r} .
$$

The $n^{\text {th }}$ moment of the reversed residual life, $M_{n}(t)=E\left[(t-X)^{n} \mid X \leq t\right]$ for $t>0$ and $n=1,2, \ldots$ uniquely determines $F(x)$. We obtain $M_{n}(t)=\frac{1}{F(t)} \int_{0}^{t}(t-x)^{n} d F(x)$. Then, the $n$th moment of the reversed residual life of $X$ becomes 


$$
M_{n}(t)=\gamma\left(1+\frac{n}{\beta},\left(\frac{1}{t}\right)^{\beta}\right)[F(t)]^{-1} \sum_{j, k, m=0}^{\infty} \sum_{r=0}^{n} \xi_{j, k, m, r}^{((2 j+k+2) \alpha, n)}, \forall n>-\beta,
$$

where

$$
\xi_{j, k, m, r}^{((2 j+k+2) \alpha, n)}=(-1)^{r} \delta_{j, k} v_{m}^{((2 j+k+2) \alpha, n)}\left(\begin{array}{l}
n \\
r
\end{array}\right) t^{n-r}
$$

\subsection{Stress-strength model}

In stress-strength modeling, $R_{X_{1}, X_{2}}=\operatorname{Pr}\left(X_{2}<X_{1}\right)$ is a measure of reliability of the system when it is subjected to random stress $X_{2}$ and has strength $X_{1}$. The system fails if and only if the applied stress is greater than its strength and the components will function satisfactorily whenever $X_{1}>X_{2} . \quad R_{X_{1}, X_{2}}$ can be considered as a measure of system performance and naturally arise in electrical and electronic systems. Other interpretation can be given as the reliability $R_{f_{1}, f_{2}}$ of a system is the probability that the system is strong enough to overcome the stress imposed on it. Let $X_{1}$ and $X_{2}$ be two independent random variables with $\mathrm{BrXEW}$ $\left(\theta_{1}, \alpha, \beta\right)$ and $\operatorname{BrXEW}\left(\theta_{2}, \alpha, \beta\right)$ distributions, respectively. The reliability is defined by $R_{f_{1}, f_{2} \mid X_{2}<X_{1}}=\int_{0}^{\infty} f_{1}\left(x ; \theta_{1}, \alpha, \beta\right) F_{2}\left(x ; \theta_{2}, \alpha, \beta\right) d x$. Then we can write

where

$$
R_{f_{1}, f_{2} \mid X_{2}<X_{1}}=\sum_{j, k, w, m=0}^{\infty} v_{j, k, w, m}
$$

$$
\begin{aligned}
& v_{j, k, w, m}=4 \theta_{1} \theta_{2} \sum \frac{(-1)^{j+w} \Gamma(2 j+k+3) \Gamma(2 w+m+3)}{j ! k ! w ! m ! \Gamma\left(\theta_{2}-h\right) \Gamma(2 j+3) \Gamma(2 w+3)} \\
& \times \sum_{i, h=0}^{\infty} \frac{(-1)^{i+h}(i+1)^{j}(h+1)^{w}\left(\begin{array}{c}
\theta_{1}-w, m=0 \\
i
\end{array}\right)\left(\begin{array}{c}
\theta_{2}-1 \\
h
\end{array}\right)}{(2 w+m+2)[(2 j+2 w+k+m+4) \alpha]} .
\end{aligned}
$$

\subsection{Order statistics}

Order statistics make their appearance in many areas of statistical theory and practice. Let $X_{1}: n, \ldots, X_{n}: n$ be a random sample from the BrXEW distribution and let $X_{(1)}, \ldots, X_{(n)}$ be the corresponding order statistics. The pdf of $i^{\text {th }}$ order statistic, say $X_{i}:{ }_{n}$, can be written as

$$
f_{i}:{ }_{n}(x)=[B(i, n-i+1)]^{-1} \sum_{j=0}^{n-i}(-1)^{j}\left(\begin{array}{c}
n-i \\
j
\end{array}\right) f(x) F^{j+i-1}(x),
$$

where $B(\cdot, \cdot)$ is the beta function. Using (3), (4), equation (12) becomes

where

$$
f(x) F(x)^{j+i-1}=\sum_{w, k=0}^{\infty} d_{w, k} \pi_{(2 w+k+2) \alpha}(x)
$$

$$
d_{w, k}=\frac{2 \theta(-1)^{w} \Gamma(2 w+k+3)}{w ! k ! \Gamma(2 w+3)[(2 w+k+2) \alpha]} \sum_{m=0}^{\infty}(-1)^{m}(m+1)^{w}\left(\begin{array}{c}
\theta(j+i)-1 \\
m
\end{array}\right) .
$$

The pdf of $X_{i: n}$ can be expressed as 


$$
f_{i: n}(x)=\sum_{w, k=0}^{\infty} \sum_{j=0}^{n-i}[B(i, n-i+1)]^{-1}(-1)^{j}\left(\begin{array}{c}
n-i \\
j
\end{array}\right) d_{w, k} \pi_{(2 w+k+2) \alpha}(x) .
$$

Then, the density function of the $i^{\text {th }}$ BrXEW order statistic is a mixture of EW densities. For example, the moments of $X_{i: n}$ can be expressed as

$$
\begin{aligned}
& E\left(X_{i: n}^{q}\right)=\sum_{w, k=0}^{\infty} \sum_{j=0}^{n-i}[B(i, n-i+1)]^{-1}(-1)^{j}\left(\begin{array}{c}
n-i \\
j
\end{array}\right) d_{w, k} \int_{-\infty}^{\infty} X_{i: n}^{q} \pi_{(2 w+k+2) \alpha}(x) d x \\
& =\Gamma\left(1+\frac{q}{\beta}\right) \sum_{w, k, m=0}^{\infty} \sum_{j=0}^{n-i} t_{w, k, m, j}^{((2 j+k+2) \alpha, q)}, \forall q>-\beta,
\end{aligned}
$$

where

$$
t_{w, k, m, j}^{((2 j+k+2) \alpha, q)}=[B(i, n-i+1)]^{-1}(-1)^{j}\left(\begin{array}{c}
n-i \\
j
\end{array}\right) d_{w, k} v_{m}^{((2 j+k+2) \alpha, q)} .
$$

\section{Parameter Estimation}

Several methods for parameter estimation were proposed in the literature but the maximum likelihood method is the most commonly employed. So, we consider the estimation of the unknown parameters of this family from complete samples only by maximum likelihood method. Let $x_{1}, \ldots, x_{n}$ be a random sample from the BrXEW distribution with parameters $\theta, \alpha$ and $\beta$. Let $\Theta=(\theta, \alpha, \beta)^{T}$ be the $3 \times 1$ parameter vector. For determining the MLE of $\Theta$, we have the log-likelihood function

$$
\begin{aligned}
& \ell=\ell(\Theta)=n \log 2+n \log \theta+n \log \alpha+n \log \beta+(\beta-1) \sum_{i=1}^{n} \log x_{i}+\sum_{i=1}^{n} \log z_{i} \\
& -\sum_{i=1}^{n} x_{i}^{\beta}+\sum_{i=1}^{n} \log z_{i}^{\alpha}-3 \sum_{i=1}^{n} \log \left(1-z_{i}^{\alpha}\right)-\sum_{i=1}^{n} s_{i}^{2}+(\theta-1) \sum_{i=1}^{n} \log \left[1-\exp \left(-s_{i}^{2}\right)\right],
\end{aligned}
$$

where $s_{i}=z_{i}^{\alpha} /\left(1-z_{i}^{\alpha}\right)$ and $z_{i}=\left[1-\exp \left(-x_{i}^{\beta}\right)\right]$. The components of the score vector can be easily obtained.

\section{Applications}

In this section, we provide two applications of the BrXEW distribution to show empirically its potentiality. In order to compare the fits of the BrXEW distribution with other competing distributions, we consider the Cramér-von Mises $\left(W^{*}\right)$ and the Anderson-Darling $\left(A^{*}\right)$ statistics. These two statistics are widely used to determine how closely a specific cdf fits the empirical distribution of a given data set. These statistics are given by

and

$$
W^{*}=\left[(1 / 12 n)+\sum_{j=1}^{n}\left[z_{j}-(2 j-1) / 2 n\right]^{2}\right](1+1 / 2 n)
$$

$$
A^{*}=\left(1+\frac{9}{4 n^{2}}+\frac{3}{4 n}\right)\left\{n+\frac{1}{n} \sum_{j=1}^{n}(-1+2 j) \log \left[z_{j}\left(1-z_{n-j+1}\right)\right]\right\}
$$

respectively, where $z_{j}=F\left(y_{j}\right)$ and the $y_{j}$ 's values are the ordered observations. The smaller these statistics are, the better the fit. The required computations are carried out using the $\mathrm{R}$ 
software. The MLEs and the corresponding standard errors (in parentheses) of the model parameters are given in Tables 1 and 2. The numerical values of the statistics $\mathrm{W}^{*}$ and $\mathrm{A}^{*}$ are listed in the same Tables. The histograms of the two data sets and the estimated pdf of the proposed model are displayed in Figures 2 and 3.

\section{Failure times of 84 aircraft windshield}

The data consist of 84 observations. Here, we shall compare the fits of the BrXEW distribution with those of other competitive models, namely: Poisson Topp Leone-Weibull (PTL-W), Marshall Olkin extended-Weibull (MOE-W) (Ghitany et al., 2005), gamma-Weibull (Ga-W) (Provost et al., 2011) Kumaraswamy-Weibull (Kw-W) (Cordeiro et al., 2010), Weibull-Fréchet (W-Fr) (Afify et al., 2016b), beta-Weibull (BW) (Lee et al., 2007), transmuted modifiedWeibull (TM-W) (Khan and King, 2013) Kumaraswamy transmuted-Weibull (KwT-W) (Afify et al., 2016a), modified beta-Weibull (MB-W) (Khan, 2015), Mcdonald-Weibull (Mc-W) (Cordeiro et al., 2014), transmuted exponentiated generalized Weibull (TExG-W) (Yousof et al., 2015) distributions, whose pdfs (for $x>0$ ) are: given by

PTL-W:

MOE-W:

$$
f(x)=\frac{2 \lambda \alpha b a^{b} x^{b-1} e^{-2 x^{b}}\left[1-\exp \left(-2 x^{b}\right)\right]^{\alpha-1}}{1-\exp (-\lambda)} \exp \left\{-\lambda\left[1-\exp \left(-2 x^{b}\right)\right]^{\alpha}\right\}
$$

GaW:

$$
f(x)=\alpha \beta \gamma^{\beta} x^{\beta-1}\left[1-(1-\alpha) e^{-(\gamma x)^{\beta}}\right]^{-2} \exp \left[-(\gamma x)^{\beta}\right]
$$

Kw-W:

$$
f(x)=\beta \alpha^{\gamma / \beta+1} \Gamma^{-1}(1+\gamma / \beta) x^{\beta+\gamma-1} \exp \left[-\alpha x^{\beta}\right]
$$

$$
f(x)=a b \beta \alpha^{\beta} x^{\beta-1}\left\{1-\exp \left[-(\alpha x)^{\beta}\right]\right\}^{a-1} \exp \left[-(\alpha x)^{\beta}\right]\left\{1-\left\{1-\exp \left[-(\alpha x)^{\beta}\right]\right\}^{a}\right\}^{b-1}
$$

W-Fr:

$$
\begin{gathered}
f(x)=a b \beta \alpha^{\beta} x^{-\beta-1}\left\{1-\exp \left[-\left(\frac{\alpha}{x}\right)^{\beta}\right]\right\}^{-b-1} \\
\times \exp \left[-b\left(\frac{\alpha}{x}\right)^{\beta}\right] \exp \left(-a\left\{\exp \left[-\left(\frac{\alpha}{x}\right)^{\beta}\right]-1\right\}^{-b}\right)
\end{gathered}
$$

B-W:

TM-W:

$$
f(x)=\beta \alpha^{\beta} B^{-1}(a, b) x^{\beta-1}\left\{1-\exp \left[-(\alpha x)^{\beta}\right]\right\}^{a-1} \exp \left[-b(\alpha x)^{\beta}\right]
$$

KT-W:

$$
f(x)=\left(\alpha+\gamma \beta x^{\beta-1}\right)\left[1-\lambda+2 \lambda \exp \left(-\alpha x-\gamma x^{\beta}\right)\right] \exp \left[-\alpha x-\gamma x^{\beta}\right]
$$

MB-W:

$$
\begin{gathered}
f(x)=a b \beta \alpha^{\beta} x^{\beta-1}\left(1+\lambda-2 \lambda\left\{1-\exp \left[-(\alpha x)^{\beta}\right]\right\}\right) \exp \left[-(\alpha x)^{\beta}\right] \\
\times\left[1-\left((1+\lambda)\left\{1-\exp \left[-(\alpha x)^{\beta}\right]\right\}-\lambda\left\{1-\exp \left[-(\alpha x)^{\beta}\right]\right\}^{2}\right)^{a}\right]^{b-1} \\
\times\left[\left\{1-\exp \left[-(\alpha x)^{\beta}\right]\right\}\left(1+\lambda-\lambda\left\{1-\exp \left[-(\alpha x)^{\beta}\right]\right\}\right)\right]^{a-1}
\end{gathered}
$$

$$
f(x)=\beta \gamma^{a} \alpha^{-\beta} B^{-1}(a, b) x^{\beta-1}\left\{1-\exp \left[-\left(\frac{x}{\alpha}\right)^{\beta}\right]\right\}^{a-1} \exp \left[-b\left(\frac{x}{\alpha}\right)^{\beta}\right]
$$


Mc-W:

$$
\times\left(1-(1-\gamma)\left\{1-\exp \left[-b\left(\frac{x}{\alpha}\right)^{\beta}\right]\right\}\right)^{-a-b}
$$

TExG-W:

$$
\begin{gathered}
f(x)=\beta c \alpha^{\beta} B^{-1}(a / c, b) x^{\beta-1}\left\{1-\exp \left[-(\alpha x)^{\beta}\right]\right\}^{a-1} \exp \left[-(\alpha x)^{\beta}\right] \\
\times\left(1-\left\{1-\exp \left[-(\alpha x)^{\beta}\right]\right\}^{c}\right)^{b-1}
\end{gathered}
$$

$$
\begin{gathered}
f(x)=a b \beta \alpha^{\beta} x^{\beta-1}\left\{1-\exp \left[-a(\alpha x)^{\beta}\right]\right\}^{b-1} \exp \left[-a(\alpha x)^{\beta}\right] \\
\times\left(1+\lambda-2 \lambda\left\{1-\exp \left[-a(\alpha x)^{\beta}\right]\right\}^{b}\right) .
\end{gathered}
$$

Some other extensions of the Weibull distribution can also be used in this comparison, but are not limited to Yousof et al., (2015), Alizadeh et al., (2016), Yousof et al., (2017a-d), Cordeiro et al., (2017a, b), Brito et al., (2017), Aryal et al., (2017a, b), Nofal et al., (2017), Korkmaz et al., (2018), Yousof et al., (2018a, b), Hamedani et al., (2018), Korkmaz et al., (2019), and Hamedani et al., (2019). The parameters of the above densities are all positive real numbers except for the TM-W and TExG-W distributions for which $|\lambda| \leq 1$. Tables 2 list the values of above statistics for seven fitted models. The MLEs and their corresponding standard errors (in parentheses) of the model parameters are also given in these tables. The figures in Table 1 reveal that the BrXEW distribution yields the lowest values of these statistics and hence provides the best fit to the two data sets. 
The Burr X Exponentiated Weibull Model: Characterizations, Mathematical Properties and Applications to Failure ...

Table 1: MLEs (standard errors in parentheses) and the statistics $W^{*}$ and $A^{*}$ for data set I.

\begin{tabular}{|c|c|c|c|}
\hline Distribution & Estimates & $W^{*}$ & $A^{*}$ \\
\hline $\operatorname{BrXEW}(\theta, \alpha, \beta)$ & $\begin{array}{c}0.6369,4.261,0.536 \\
(0.356),(1.757),(0.099)\end{array}$ & 0.074 & 0.64 \\
\hline PTL-W $(\lambda, \alpha, b)$ & $\begin{array}{c}-5.78175,4.22865,0.65801 \\
(1.395),(1.167),(0.039)\end{array}$ & 0.1397 & 1.194 \\
\hline $\operatorname{MOE}-\mathrm{W}(\gamma, \beta, \alpha)$ & $\begin{array}{c}488.899455,0.283246,1261.9660 \\
(189.358),(0.013),(351.073)\end{array}$ & 0.3995 & 4.448 \\
\hline $\mathrm{Ga}-\mathrm{W}(\alpha, \beta, \gamma)$ & $\begin{array}{l}2.376973,0.848094,3.534401 \\
(0.378),(0.0005296),(0.665)\end{array}$ & 0.255 & 1.9488 \\
\hline $\mathrm{Kw}-\mathrm{W}(\alpha, \beta, a, b)$ & $\begin{array}{c}14.4331,0.2041,34.6599,81.8459 \\
(27.095),(0.042),(17.527),(52.014)\end{array}$ & 0.1852 & 1.5059 \\
\hline $\mathrm{W}-\operatorname{Fr}(\alpha, \beta, a, b)$ & $\begin{array}{c}630.9384,0.3024,416.0971,1.1664 \\
(697.94),(0.03),(232.36),(0.357)\end{array}$ & 0.25372 & 1.957 \\
\hline $\mathrm{B}-\mathrm{W}(\alpha, \beta, a, b)$ & $\begin{array}{l}1.36,0.2981,34.1802,11.4956 \\
(1.002),(0.06),(14.838),(6.73)\end{array}$ & 0.4652 & 3.2197 \\
\hline $\mathrm{TM}-\mathrm{W}(\alpha, \beta, \gamma, \lambda)$ & $\begin{array}{c}0.2722,1,4.6 \times 10^{-6}, 0.4685 \\
(0: 014),\left(5.2 \times 10^{-5}\right),\left(1.9 \times 10^{-4}\right),(0.165)\end{array}$ & 0.80649 & 11.2047 \\
\hline $\mathrm{KwT}-\mathrm{W}(\alpha, \beta, \lambda, a, b)$ & $\begin{array}{c}27.7912,0.178,0.4449,29.5253,168.0603 \\
(33.401),(0.017),(0.609),(9.792),(129.165)\end{array}$ & 0.164 & 1.363 \\
\hline $\mathrm{MB}-\mathrm{W}(\alpha, \beta, a, b, c)$ & $\begin{array}{c}10.1502,0.1632,57.4167,19.3859,2.0043 \\
(18.697),(0.019),(14.063),(10.019),(0.662)\end{array}$ & 0.47172 & 3.26561 \\
\hline $\operatorname{Mc}-\mathrm{W}(\alpha, \beta, a, b, c)$ & $\begin{array}{l}\text { 1.9401, 0.306, 17.686, 33.6388, 16.7211, } \\
(1.011),(0: 045),(6.222),(19.994),(9.722)\end{array}$ & 0.1986 & 1.5906 \\
\hline TExG-W $(\alpha, \beta, \lambda, a, b)$ & $\begin{array}{c}4.2567,0.1532,0.0978,5.2313,1173.3277 \\
(33.401),(0: 017),(0.609),(9.792)\end{array}$ & 1.0079 & 6.233 \\
\hline
\end{tabular}




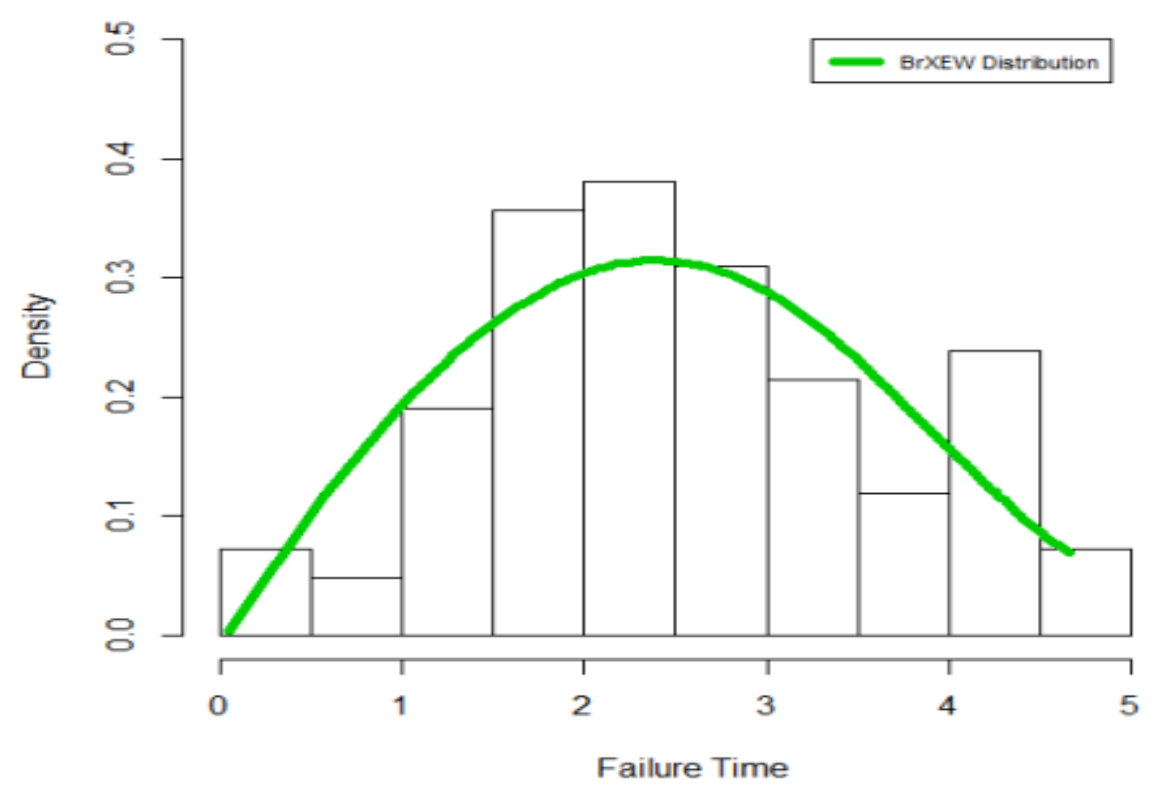

Figure 3: Estimated pdf for data set I.

\section{Survival times (in days) of $\mathbf{7 2}$ Guinea pigs}

The second real data set corresponds to the survival times (in days) of 72 guinea pigs infected with virulent tubercle bacilli reported by Bjerkedal (1960). We shall compare the fits of the BrXEW distribution with those of other competitive models, namely: Weibull-Weibull (W-W) (Tahir et al., 2016), odd Weibull-Weibull (OW-W) (Bourguignon et al., 2014),Weibull LogWeibull (WLog-W) (Alzaatreh et al., 2013), the gamma exponentiated-exponential (GaE-E) (Risti'c and Balakrishnan 2012) and exponential exponential-geometric (EE-Gc) (Rezaei et al., 2013) distributions, whose pdfs (for $x>0$ ) are: given by W-W:

OW-W:

$$
f(x)=\exp \left(-\alpha\left\{-\log \left[1-\exp \left(-\lambda x^{\gamma}\right)\right]\right\}^{\beta}\right) ;
$$

GaE-E:

$$
f(x)=1-\exp \left\{-\alpha\left[\exp \left(\lambda x^{\gamma}\right)-1\right]^{\beta}\right\}
$$

$$
f(x)=\frac{\alpha \theta}{\Gamma(\lambda)} \exp (-\theta x)[1-\exp (-\theta x)]^{\alpha-1}\{-\alpha \log [1-\exp (-\theta x)]\}^{\lambda-1}
$$

EE-Gc:

$$
f(x)=\frac{\alpha \theta(1-p) \exp (-\theta x)}{[1-\exp (-\theta x)]^{\alpha-1}\left\{1-p+p[1-\exp (-\theta x)]^{\alpha}\right\}^{2}} .
$$


Table 2: MLEs (standard errors in parentheses) and the statistics $\mathrm{W}^{*}$ and $\mathrm{A}^{*}$ for data set II.

\begin{tabular}{lc|cc}
\hline \hline Distribution & Estimates & $W^{*}$ & $A^{*}$ \\
\hline $\operatorname{BrXEW}(\theta, \alpha, \beta)$ & $3.18,5.539,0.166$ & $\mathbf{0 . 0 9 0 7}$ & $\mathbf{0 . 5 6 6 8}$ \\
& $(2.117),(2.437),(0.024)$ & & \\
$\mathrm{W}-\mathrm{W}(\beta, \gamma, \lambda)$ & $2.6594,0.6933,0.0270$ & 0.1427 & 0.7811 \\
& $(0.713),(0.1707),(0.019)$ & & \\
OW-W $(\beta, \gamma, \lambda)$ & $11.1576,0.0881,0.4574$ & 0.4494 & 2.4764 \\
& $(4.545)(0.0355)(0.077)$ & & \\
$\mathrm{WLog}-\mathrm{W}(\beta, \gamma, \lambda)$ & $1.7872,0.7795,0.0255$ & 0.4348 & 2.3938 \\
& $(0.782),(0.333),(0.040)$ & & \\
$\operatorname{GaE}-\mathrm{E}(\lambda, \alpha, \theta)$ & $2.1138,2.6006,0.0083$ & 0.3150 & 1.7208 \\
$\operatorname{EE}-\mathrm{Gc}(\alpha, \theta, p)$ & $(1.3288),(0.559),(0.005)$ & & \\
& $2.5890,0.0004,0.9999$ & 0.1047 & 0.5789 \\
\hline \hline
\end{tabular}

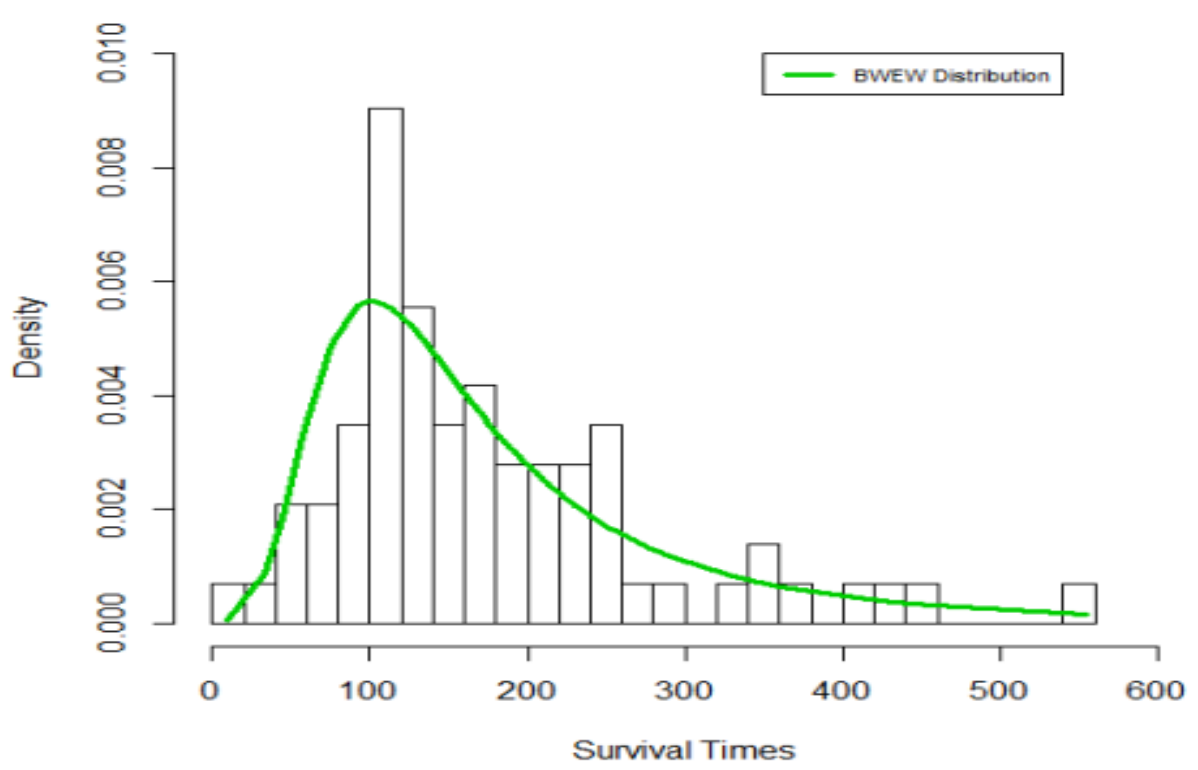

Figure 4: Estimated pdf for data set II.

Based on the figures in Tables 1 and 2 we conclude that the BrXEW lifetime model provides adequate fits as compared to other Weibull-G models in both applications with small values for $\mathrm{W}^{*}$ and $\mathrm{A}^{*}$. In Application 1, the proposed BrXEW lifetime model is much better than the PTLW, MOE-W, Ga-W, Kw-W, W-Fr, B-W, TM-W, KwT-W, MB-W, Mc-W, TExG-W models, 
and a good alternative to these models. In Application 2, the proposed BrXEW lifetime model is much better than the W-W, OW-W, WLog-W, GaE-E, EE-Gc models, and a good alternative to these models.

\section{Conclusions}

In this article, we introduce a new three-parameter lifetime model called the Burr $\mathrm{X}$ exponentiated Weibull model. The major justification for the practicality of the new lifetime model is based on the wider use of the exponentiated Weibull and Weibull models. We are motivated to propose this new lifetime model because it exhibits increasing, decreasing, bathtub, J shaped and constant hazard rates. The new lifetime model can be viewed as a mixture of the exponentiated Weibull distribution. It can also be viewed as a suitable model for fitting the right skewed, symmetric, left skewed and unimodal data. We provide a comprehensive account of some of its statistical properties also some useful characterization results are presented. The maximum likelihood method is used to estimate the model parameters. We prove empirically the importance and flexibility of the new model in modeling two types of lifetime data. The proposed BrXEW lifetime model is a much better fit than the Poisson Topp LeoneWeibull, the Marshall Olkin extended-Weibull, gamma-Weibull, Kumaraswamy-Weibull, Weibull-Fréchet, beta-Weibull, transmuted modified-Weibull, Kumaraswamy transmutedWeibull, modified beta-Weibull, Mcdonald-Weibull and transmuted exponentiated generalized-Weibull models, so the new lifetime model is a good alternative to these models in modeling aircraft windshield data. It is also a much better fit than the Weibull-Weibull, odd Weibull-Weibull, Weibull Log-Weibull, the gamma exponentiated-exponential and exponential exponential-geometric models, so it is a good alternative to these models in modeling the survival times of Guinea pigs. We hope that the new model will attract wider applications in reliability, engineering and other areas of research.

\section{References}

1. Afify, A. Z., Cordeiro, G. M., Yousof, H. M. Alzaatreh, A., and Nofal, Z. M. (2016a). The Kumaraswamy transmuted-G family of distributions: properties and applications, 14, 245-270.

2. Afify, A. Z., Yousof, H. M., Cordeiro,G. M., Ortega, E. M. M. and Nofal, Z. M. (2016b). The Weibull Fréchet distribution and its applications, Journal of Applied Statistics, 43, 2608--2626.

3. Alizadeh, M., Yousof, H. M., Afify, A. Z., Cordeiro, G.M. and Mansoor, M. (2016). The complementary generalized transmuted Poisson-G family of distributions. Austrian Journal of Statistics, forthcoming.

4. Alzaatreh, A., Lee, C. and Famoye, F. (2013). A new method for generating families of continuous distributions, Metron, 71, 63-79.

5. Aryal, G. R., Ortega, E. M., Hamedani, G. G. and Yousof, H. M. (2017a). The ToppLeone Generated Weibull distribution: regression model, characterizations and applications, International Journal of Statistics and Probability, 6, 126-141.

6. Aryal, G. R. and Yousof, H. M. (2017b). The exponentiated generalized-G Poisson family of distributions. Economic Quality Control, 32(1), 1-17.

7. Bjerkedal, T. (1960). Acquisition of resistance in guinea pigs infected with different doses of virulent tubercle bacilli. American Journal of Hygiene, 72, 130-148.

8. Bourguignon, M., Silva, R.B. and Cordeiro, G.M. (2014). The Weibull-G family of probability distributions, Journal of Data Science 12, 53-68. 
9. Brito, E., Cordeiro, G. M., Yousof, H. M., Alizadeh, M. and Silva, G. O. (2017). ToppLeone Odd Log-Logistic Family of Distributions, Journal of Statistical Computation and Simulation, 87(15), 3040-3058.

10. Cordeiro, G. M., Afify, A. Z., Yousof, H. M., Pescim, R. R. and Aryal, G. R. (2017a). The exponentiated Weibull-H family of distributions: Theory and Applications. Mediterranean Journal of Mathematics, 14, 1-22.

11. Cordeiro, G.M., Afify, A. Z., Yousof, H. M., Cakmakyapan, S. and Ozel, G. (2018). The Lindley Weibull distribution: properties and applications, Anais da Academia Brasileira de Ciências, forthcoming.

12. Cordeiro, G. M., Hashimoto, E. M., Edwin, E. M. M. Ortega. (2014). The McDonald Weibull model. Statistics: A Journal of Theoretical and Applied Statistics, 48, 256--278.

13. Cordeiro, G. M., Ortega, E. M. and Nadarajah, S. (2010). The Kumaraswamy Weibull distribution with application to failure data. Journal of the Franklin Institute, 347, 13991429.

14. Cordeiro, G. M., Yousof, H. M., Ramires, T. G. and Ortega, E. M. M. (2017b). The Burr XII system of densities: properties, regression model and applications. Journal of Statistical Computation and Simulation, 88(3), 432-456.

15. Glänzel, W. (1987). A characterization theorem based on truncated moments and its application to some distribution families, Mathematical Statistics and Probability Theory (Bad Tatzmannsdorf, 1986), Vol. B, Reidel, Dordrecht, 75-84.

16. Glänzel, W. (1990). Some consequences of a characterization theorem based on truncated moments, Statistics: A Journal of Theoretical and Applied Statistics, 21 (4), 613-618.

17. Hamedani, G. G. (2013). On certain generalized gamma convolution distributions II, Technical Report No. 484, MSCS, Marquette University.

18. Hamedani, G. G. Yousof, H. M., Rasekhi, M., Alizadeh, M., Najibi, S. M. (2018). Type I general exponential class of distributions. Pak. J. Stat. Oper. Res. forthcoming.

19. Hamedani, G. G. Rasekhi, M., Najibi, S. M., Yousof, H. M. and Alizadeh, M. (2019). Type II general exponential class of distributions. Pak. J. Stat. Oper. Res., forthcoming.

20. Khan, M. N. (2015). The modied beta Weibull distribution. Hacettepe Journal of Mathematics and Statistics, 44, 1553-1568.

21. Khan, M. S. and King, R. (2013). Transmuted modied Weibull distribution: a generalization of the modied Weibull probability distribution. European Journal of Pure and Applied Mathematics, 6, 66-88.

22. Kilbas, A. A., Srivastava, H. M. and Trujillo, J. J. (2006). Theory and Applications of Fractional Difierential Equations. Elsevier, Amsterdam.

23. Korkmaz, M. C. Yousof, H. M., Rasekhi, M. Hamedani G. G. (2018). The exponential Lindley odd log-logistic $G$ family: properties, characterizations and applications. Journal of Statistical Theory and Applications, forthcoming.

24. Korkmaz, M. C., Altun, E., Yousof, H. M. and Hamedani G. G. (2019). The Odd Power Lindley Generator of Probability Distributions: Properties, Characterizations and Regression Modeling, International Journal of Statistics and Probability, 8(2), 70-89.

25. Lee, C., Famoye, F. and Olumolade, O. (2007). Beta-Weibull distribution: some properties and applications to censored data. Journal of Modern Applied Statistical Methods, 6, 17.

26. Mudholkar, G. S. and Srivastava, D. K. (1993). Exponentiated Weibull family for analyzing bathtub failure-rate data. IEEE Transactions on Reliability, 42, 299-302.

27. Mudholkar, G. S., Srivastava, D. K. and Freimer, M. (1995). The exponentiated Weibull 
family: A reanalysis of the bus-motor-failure data. Technometrics, 37, 436-445.

28. Murthy, D. N. P., Xie, M. and Jiang, R. (2004). Weibull Models. John Wiley and Sons, Hoboken, New Jersey.

29. Nadarajah, S., Cordeiro, G. M. and Ortega, E. M. M. (2013). The exponentiated Weibull distribution: A survey, Statistical Papers, 54, 839-877.

30. Nofal, Z. M., Afify, A. Z., Yousof, H. M. and Cordeiro, G. M. (2017). The generalized transmuted-G family of distributions. Communications in Statistics-Theory and Methods, 46, 4119-4136.

31. Provost, S.B. Saboor, A. and Ahmad, M. (2011). The gamma--Weibull distribution, Pak. Journal Stat., 27, 111-131.

32. Rezaei, S., Nadarajah, S. and Tahghighnia, N. A (2013). new three-parameter lifetime distribution, Statistics, 47, 835-860.

33. Rinne, H. (2009). The Weibull Distribution: A Handbook. CRC Press, Boca Raton, Florida.

34. Ristic, M.M. and Balakrishnan, N. (2012). The gamma-exponentiated exponential distribution, Journal of Statistical Computation and Simulation, 82, 1191-1206.

35. Yousof, H. M., Afify, A. Z., Alizadeh, M., Butt, N. S., Hamedani, G. G. and Ali, M. M. (2015). The transmuted exponentiated generalized-G family of distributions. Pak. J. Stat. Oper. Res., 11, 441-464.

36. Yousof, H. M., Afify, A. Z., Alizadeh, M., Nadarajah, S., Aryal, G. R. and Hamedani, G. G. (2018a). The Marshall-Olkin generalized-G family of distributions with Applications, STATISTICA, 78(3), 273- 295.

37. Yousof, H. M., Afify, A. Z., Cordeiro, G. M. Alzaatreh, A. and Ahsanullah, M. (2017a). A new four-parameter Weibull model, Journal of Statistical Theory and Applications, 16(4), $448-466$.

38. Yousof, H. M., Afify, A. Z., Hamedani, G. G. and Aryal, G. (2017b). The Burr X generator of distributions for lifetime data. Journal of Statistical Theory and Applications, 16, 288-305.

39. Yousof, H. M., Alizadeh, M., Jahanshahiand, S. M. A., Ramires, T. G., Ghosh, I. and Hamedani G. G. (2017c). The transmuted Topp-Leone G family of distributions: theory, characterizations and applications, Journal of Data Science. 15, 723-740.

40. Yousof, H. M., Majumder, M., Jahanshahi, S. M. A., Ali, M. M. and Hamedani G. G. (2018b). A new Weibull class of distributions: theory, characterizations and applications, Journal of Statistical Research of Iran, 15, 45-83.

41. Yousof, H. M., Rasekhi, M., Afify, A. Z., Alizadeh, M., Ghosh, I. and Hamedani G. G. (2017d). The beta Weibull-G family of distributions: theory, characterizations and applications, Pakistan Journal of Statistics, 33, 95-116. 